
hypertension but does not cause its development

BMY Cheung, KL Ong, AWK Tso, RYH Leung, SS Cherny, PC Sham, GN Thomas, TH Lam, KSL Lam Department of Medicine, The University of Hong Kong, Hong Kong

Introduction: Interleukin-6 (IL-6) plays a central role in inflammation and insulin resistance as well as atherogenesis. We investigated the associations of plasma IL-6 and its genetic variants with hypertension in both cross-sectional and prospective study designs.

Methods: Plasma IL-6 was measured in 648 normotensive and 294 hypertensive subjects from the Hong Kong Cardiovascular Risk Factor Prevalence Study-2 (CRISPS-2) in 2000-2004 and three tagging SNPs in the IL-6 gene (IL6) were genotyped. Among subjects normotensive in CRISPS-2, 515 subjects were followed up in CRISPS-3 in 20052008 and 100 of them had developed hypertension.

Results: Plasma IL-6 correlated with systolic blood pressure $(r=0.128, \mathrm{P}<0.001)$, pulse pressure $(r=0.144, \mathrm{P}<0.001)$, and mean arterial pressure $(r=0.086, \mathrm{P}=0.008)$. Hypertensive subjects have significantly higher plasma IL-6 level after adjusting for age and sex (geometric mean $[95 \% \mathrm{Cl}]=0.60[0.54-0.65]$ vs $0.47[0.44-0.50] \mathrm{pg} / \mathrm{mL}, \mathrm{P}=0.021)$. In stepwise logistic regression, plasma IL-6 was associated with hypertension in women ( $\mathrm{P}=0.004)$, but not in men. The SNP rs1800796 was associated with plasma IL-6 (beta $=-0.098, \mathrm{P}=0.002)$ in stepwise linear regression. However, this SNP was not associated with hypertension or blood pressure. Among subjects normotensive in CRISPS-2, plasma IL-6 was not associated with the development of hypertension in CRISPS-3.

Conclusion: Elevated plasma IL-6 is associated with hypertension, especially in women. Plasma IL-6 is influenced by the SNP rs1800796. However, this SNP is not associated with hypertension, suggesting that hypertension is caused by other factors that elevate plasma IL-6.

Acknowledgement: This study was funded by Hong Kong Research Grant Council grants (HKU7229/01M and HKU7626/07M) and the Sun Chieh Yeh Heart Foundation.

\title{
Association of a genetic variant in the apolipoprotein A5 gene with the metabolic syndrome
} in Chinese

BMY Cheung, KL Ong, CQ Jiang, B Liu, YL Jin, AWK Tso, S Tam, KS Wong, B Tomlinson, JM Lin, XJ Yue, KSL Lam, TH Lam, GN Thomas

Department of Medicine, The University of Hong Kong, Hong Kong

Introduction: We previously reported that the single nucleotide polymorphism (SNP) rs662799 $(-1131 \mathrm{~T}>\mathrm{C})$ in the apolipoprotein A5 gene (APOA5) was an important determinant of plasma triglycerides in both Hong Kong and Guangzhou Chinese. We, therefore, investigated the association of SNPs in APOA5 with the metabolic syndrome (MetS) in the Hong Kong and Guangzhou Chinese.

Methods: MetS was defined according to the consensus criteria proposed jointly by several organisations in 2009. Five tagging SNPs were genotyped in 1330 unrelated subjects from the Hong Kong Cardiovascular Risk Factor Prevalence Study cohort with follow-up after a median interval of 6.4 years. A total of 1952 subjects from the Guangzhou Biobank Cohort Study-Cardiovascular Disease Subcohort were used to replicate the findings.

Results: The minor allele of rs662799 was significantly associated with higher odds for the MetS in Hong Kong subjects at both baseline $(\mathrm{OR}=1.47, \mathrm{P}=0.00082)$ and follow-up $(\mathrm{OR}=1.30, \mathrm{P}=0.010)$. A similar association was found in Guangzhou subjects $(\mathrm{OR}=1.27, \mathrm{P}=0.0041)$. In a pooled sample of Hong Kong subjects at follow-up and Guangzhou subjects, this SNP was also associated with HDL and LDL cholesterol $(\mathrm{P}<0.001$ and 0.010 , respectively). All these associations disappeared after further adjusting for plasma triglycerides $(\mathrm{P}>0.05)$. In a meta-analysis of six studies, the combined OR $(95 \% \mathrm{Cl})$ was 1.38 (1.25-1.52) for the TC + CC genotype compared with the TT genotype $(\mathrm{P}<0.00001)$.

Conclusion: The association of $-1131 \mathrm{~T}>\mathrm{C}$ polymorphism in APOA5 with the MetS was mainly due to its strong effect on plasma triglycerides. Further studies are needed to assess the utility of this genetic marker in risk stratification.

Acknowledgement: This study was funded by Hong Kong Research Grant Council grants (HKU7229/01M and HKU7626/07M), the Sun Chieh Yeh Heart Foundation and a grant from the National Natural Science Foundation of China/Research Grants Council of Hong Kong Joint Research Scheme (30518001/CO301070202 and HKU720/05). 